\title{
Left Hemisphere Lateralization of Epileptic Focus Can Be More Frequent in Temporal Lobe Epilepsy Surgical Patients with No Consensus Associated with Depression Lateralization
}

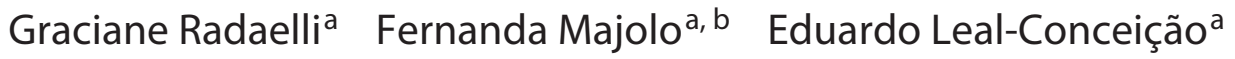 \\ Francisco de Souza Santos ${ }^{a}$ Vinícius Escobar ${ }^{a}$ Gabriele Goulart Zanirati ${ }^{a}$ \\ Mirna Wetters Portuguez ${ }^{a, c}$ Fulvio Alexandre Scorza ${ }^{d, e}$ Jaderson Costa da Costa ${ }^{a, c, e}$ \\ aBrain Institute of Rio Grande do Sul (Bralns), Pontifical Catholic University of Rio Grande do Sul, Porto Alegre, Brazil; \\ bPost-graduate Program in Biotechnology, Universidade do Vale do Taquari - Univates, Lajeado, Brazil; 'School of Medicine, \\ Pontifical Catholic University of Rio Grande do Sul, Porto Alegre, Brazil; dDepartment of Neurology and Neurosurgery,

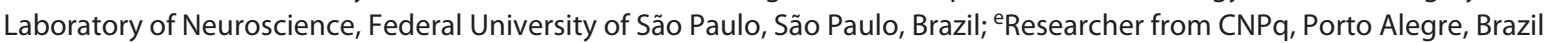

\section{Keywords}

Anxiety · Depression · Epilepsy · Epileptogenic focus ·

Systematic review

\begin{abstract}
Temporal lobe epilepsy (TLE) is considered to be the most common form of epilepsy, and it has been seen that most patients are refractory to antiepileptic drugs. A strong association of this ailment has been established with psychiatric comorbidities, primarily mood and anxiety disorders. The side of epileptogenic may contribute to depressive and anxiety symptoms; thus, in this study, we performed a systematic review to evaluate the prevalence of depression in TLE in surgical patients. The literature search was performed using PubMed/Medline, Web of Science, and PsycNet to gather data from inception until January 2019. The search strategy was related to TLE, depressive disorder, and anxiety. After
\end{abstract}

reading full texts, 14 articles meeting the inclusion criteria were screened. The main method utilized for psychiatric diagnosis was Diagnostic and Statistical Manual of Mental Disorders/Structured Clinical Interview for DSM. However, most studies failed to perform the neuropsychological evaluation. For those with lateralization of epilepsy, focus mostly occurred in the left hemisphere. For individual depressive diagnosis, 9 studies were evaluated, and 5 for anxiety. Therefore, from the data analyzed in both situations, no diagnosis was representative in preoperative and postoperative cases. In order to estimate the efficacy of surgery in the psychiatry episodes and its relation to seizure control, the risk of depression and anxiety symptoms in epileptic patients need to be determined before surgical procedures. Rigorous preoperative and postoperative evaluation is essential for psychiatry conditions in patients with refractory epilepsy candidates for surgery.

(c) 2021 S. Karger AG, Basel karger@karger.com

(c) 2021 S. Karger AG, Basel

www.karger.com/dne

Karger ${ }^{\prime}=$
Jaderson Costa da Costa

Instituto do Cérebro RS, Pontifícia Universidade Católico do RS - PUCRS Ipiranga, 6690

Porto Alegre 90610-000 (Brazil)

jcc@pucrs.br 
Fig. 1. Summary of evidence search and

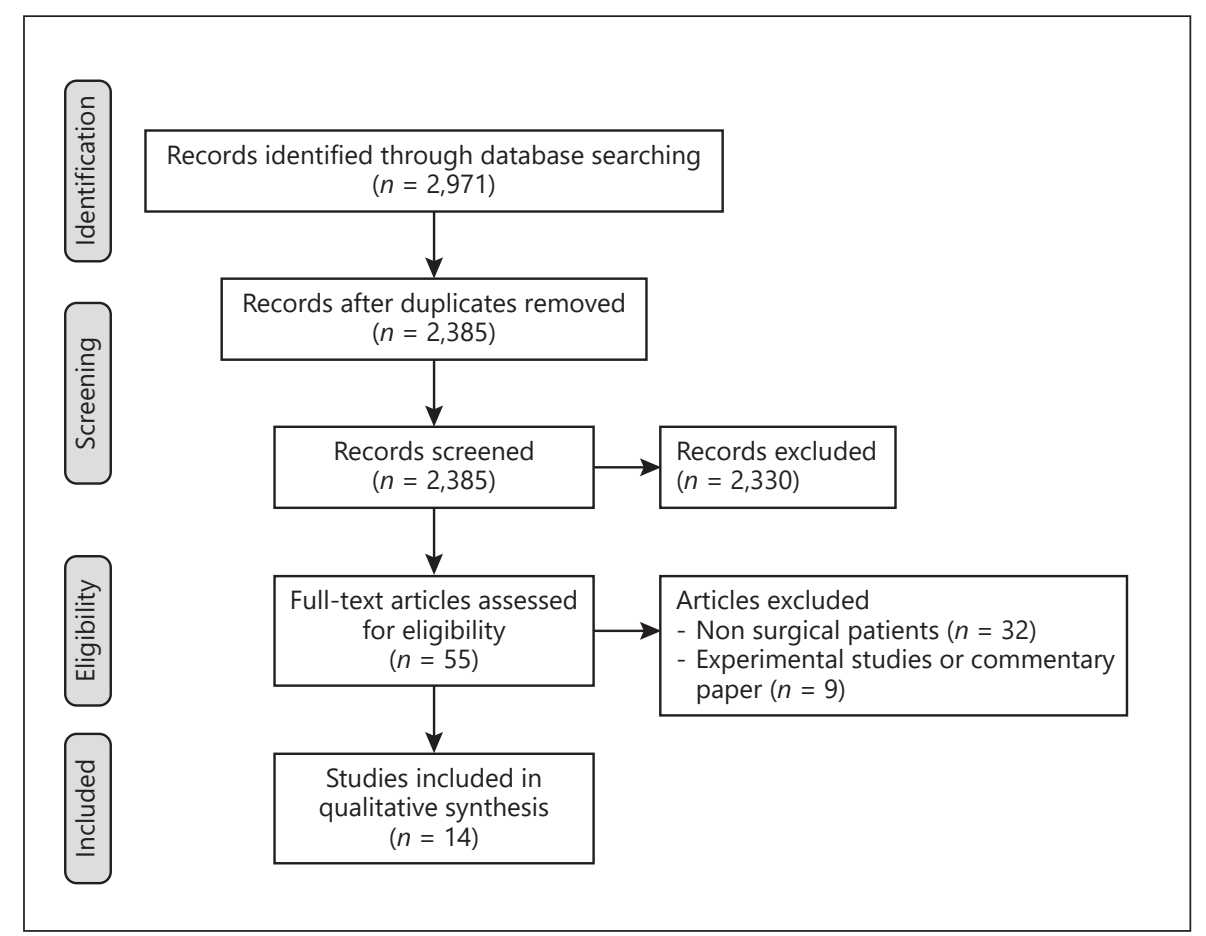
study selection.

\section{Introduction}

The most common form of focal epilepsy is temporal lobe epilepsy (TLE). Most of the patients are refractory to antiepileptic drugs (AEDs), about $75 \%$ presenting resistance to medical treatment [1-3]. Psychiatric comorbidities, such as anxiety disorders, are often expressed among patients with TLE [4-7]. The exact mechanism behind this association remains to be explored; however, the major findings indicate that these comorbidities and TLE share similar neuroanatomic localization $[3,8]$. Another factor that remains uncertain and may contribute to anxiety and depressive symptoms in TLE is the side of the epileptogenic focus. Research has documented the prevalence of interictal depression in left-sided seizure foci $[9,10]$, while others illustrate a tendency for greater depressive symptoms in presurgical right-sided seizure foci patients $[11,12]$.

Depressive and anxiety symptoms in epilepsy patients are evaluated by the scales and inventories established. A quantitative measure of recurrent and severity of mood symptomatology experienced by them can be recorded by these tests $[13,14]$. Neuropsychological functions in TLE patients can also be influenced by antagonist effects such as underlying injury, mood disorders, and AEDs treatment. In this regard, neuropsychological assessments are substantial to comprehend better the impact of those variables on the lives of patients. The interaction between laterality of TLE, usage of AEDs, depressive symptoms, and neuropsychological functions remains to be elucidated. For a better comprehension of this association, we proposed a systematic and comprehensive literature review searching for the outcomes of the correlation between these aspects in surgical patients.

\section{Methods}

A systematic review was conducted using the methodology outlined in the Cochrane Handbook for Systematic Reviewers [15]. The data were reported following the Preferred Reporting Items for Systematic Reviews and Meta-Analyses [16]. The review protocol was registered under the number CRD42019104443 in the International Register of Prospective Systematic Reviews.

\section{Database Search}

A literature search was performed in PubMed/Medline, Web of Science, and PsycNet to obtain data from inception until January 2019. The following keywords and medical subject headings (MeSH) were used in the search strategy: "epilepsy," "temporal lobe" OR "epilepsy” AND "temporal” AND "lobe” OR "temporal lobe epilepsy" OR "temporal" AND "lobe” AND "epilepsy” AND "depressive disorder" OR "depressive" AND "disorder" OR "depressive disorder" OR "depression" OR "depression" OR "anxiety" OR "anxiety." The strategies for other databases are available on request. Articles published in all languages were included. The bibliography of the included articles was screened manually. The titles 
Table 1. Clinical characteristics of surgical patients with TLE related to the diagnosis and treatment

\begin{tabular}{lc}
\hline Study & $N(\%)$ \\
\hline Sample $(n=14)$ & \\
Mean (SD) & $103.14( \pm 107.44)$ \\
Design & $3(21.4)$ \\
Cross-sectional follow-up & $6(28.5)$ \\
Prospective & $4(28.5)$ \\
Retrospective & $1(7.14)$ \\
Cohort & $12(85.7)$ \\
Trail & $2(14.2)$ \\
Single-center & $5(35.7)$ \\
Multi-center & $2(14.3)$ \\
Method of PD & $7(50)$ \\
DSM/SCID & \\
CIDI & $4(28.5)$ \\
None & $10(71.5)$ \\
Neuropsychological evaluation & \\
Yes & 457 \\
No & 483 \\
Lateralization of epileptic focus $(n=11)$ & 2 \\
Right & $6(42.8)$ \\
Left & $8(57.2)$ \\
Bilateral & \\
Use of AEDs & \\
Reported & \\
Not reported & \\
\hline
\end{tabular}

DSM, Diagnostic and Statistical Manual of Mental Disorders; SCID, Structured Clinical Interview for DSM; CIDI, Composite International Diagnostic Interview; AEDs, antiepileptic drugs; TLE, temporal lobe epilepsy; PD, psychiatric diagnosis.

and abstracts of all studies identified in the search based on the abovementioned terms and MeSH were evaluated independently by 2 authors (E.L.C. and F.S.S.). Disagreements were resolved by consensus or by a third reviewer (G.R.).

\section{Eligibility Criteria}

The inclusion criteria for the present review were as follows: articles without language restriction; diagnosis of epilepsy by electrocardiogram or magnetic resonance imaging; psychiatric diagnosis (PD) (by scales, interviews, diagnostic manuals - Diagnostic and Statistical Manual of Mental Disorders [DSM] and ICD-10); study design (case series with $>10$ patients, retrospective and prospective, clinical, human); unilateral or bilateral TLE; surgical patients. Studies of systematic reviews, letters, and experimental studies; children under 16 years; patients with dysphoria; patients with generalized epilepsy (multiple foci); non-surgical patients were the exclusion criteria. Figure 1 displayed a flowchart of study selection and inclusion.

\section{Data Extraction}

The databases were investigated, and duplicate entries were eliminated. The full-text articles whose abstracts did not provide sufficient information regarding the inclusion and exclusion crite-
Table 2. Clinical characteristics related to the lateralization of epileptic focus and depression and anxiety symptoms of surgical patients with TLE

\begin{tabular}{lccc}
\hline \multicolumn{4}{c}{ Individual depressive diagnosis } \\
\cline { 2 - 4 } & preoperative & postoperative & $\chi^{2}$ ( $p$ value $)$ \\
\hline $\begin{array}{l}\text { Studies - depression } \\
\text { Yes }\end{array}=9$ studies $)$ & & \\
No & 221 & 111 & $<0.001$ \\
773 & 883 & 0.006 \\
$\begin{array}{l}\text { Studies - anxiety }(n=5 \text { studies }) \\
\text { Yes }\end{array}$ & & \\
No & 122 & 86 & \\
\hline
\end{tabular}

TLE, temporal lobe epilepsy.

ria were selected for evaluation. In the second phase, the same reviewers independently evaluated the full text of these articles and made their selection based on the eligibility criteria. Data on the following were collected: the number of patients, method of PD, symptoms diagnosis criteria, diagnostic scales, neuropsychological tests, report of the number of AEDs, lateralization of the epileptic focus, individual depression symptoms, lateralized depression symptoms, individual anxiety symptoms, lateralized anxiety symptoms, and the design/classification of evidence.

\section{Results}

The search retrieved 2,971 potentially relevant citations from the electronic databases. By removing duplicate titles, 55 articles were eliminated. After screening titles and abstracts, only 14 articles met the inclusion criteria (Fig. 1). The clinical design approach included prospective $(28.5 \%)$, retrospective $(28.5 \%)$, cross-sectional follow-up (21.4\%), and cohort (7.14\%). Most of them were single-center studies (85.7\%).

Overall, 14 surgical studies with TLE were evaluated, and DSM/Structured Clinical Interview for DSM was the main method used for PD (35.7\%). However, about $50 \%$ of the studies did not employ any kind of PD. Moreover, the neuropsychological evaluation was not explored by most of the studies (71.5\%). From 11 studies that portrayed clinical cases of lateralization of the epileptic focus, most of them were found to occur in the left hemisphere (483 patients) (Table 1). Nine studies were evaluated for individual depressive diagnosis and 5 to anxiety; therefore, from the data analyzed in both situations, no diagnosis was representative in preoperative and postoperative cases $(p<0.05)$ (Table 2$)$. Regarding the types of treatment using AED, most studies 
Table 3. Clinical characteristics related to the diagnosis and treatment of surgical patients with TLE

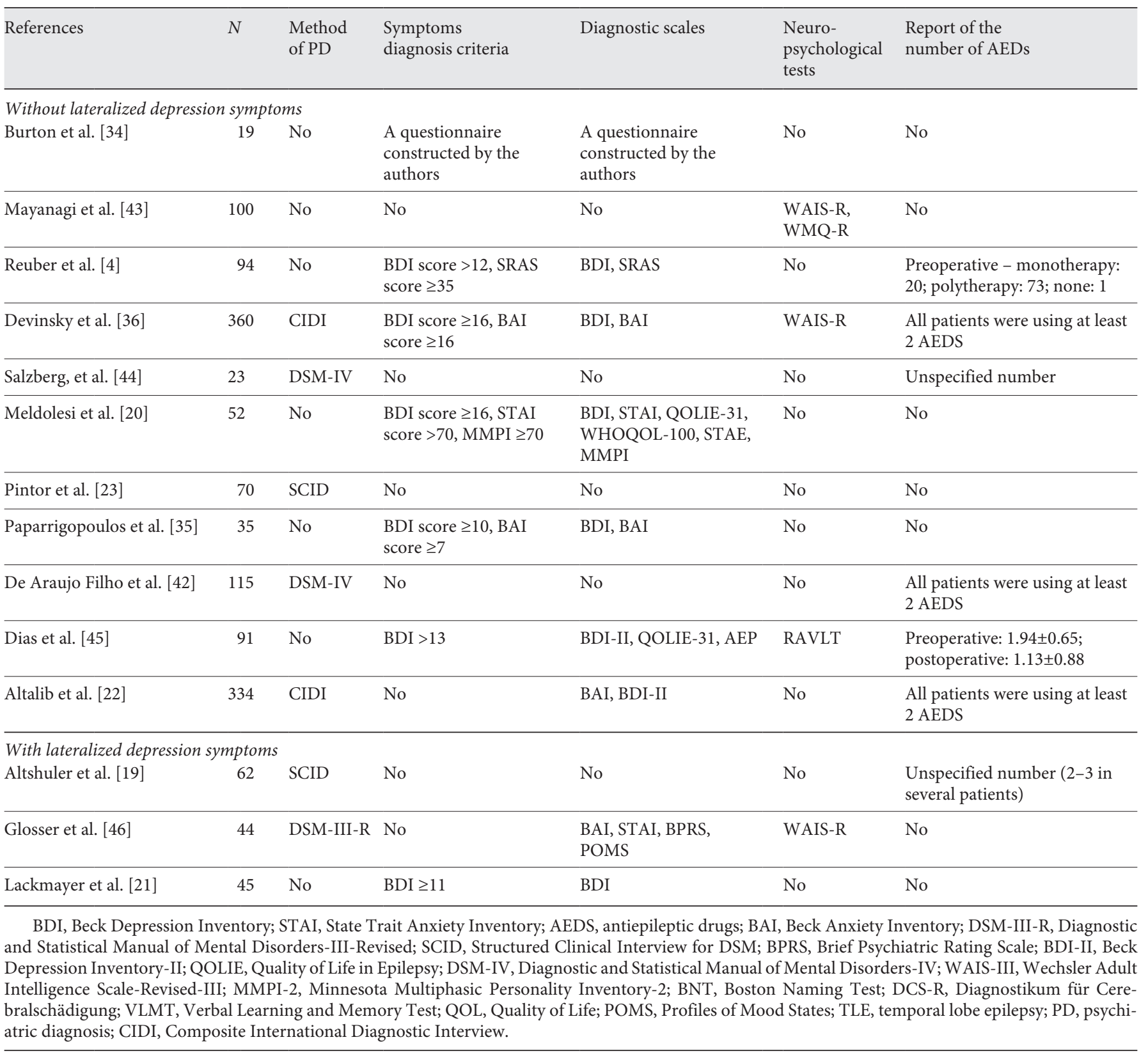

failed to report the treatment utilized (57.2\%). More details about each study evaluated above are summarized in Tables 3 and 4.

\section{Discussion}

Several beneficial outcomes, such as reduced seizure frequency, improved quality of life, and mood dysfunction, have been known to be associated with surgical pro- cedures for treating refractory epilepsy $[17,18]$. Temporal lobectomy could be a resolution for depression and anxiety symptoms in epileptic patients since the limbic system plays a crucial role in increasing the risk of these conditions [19]. Studies documented that for the patients with seizure focus in the temporal lobe, depression symptoms are more frequent as compared to those with an epileptic focus in extratemporal lobe regions, and the epilepsy surgery could resolve the depression symptoms in some of the patients [19]. Reduction in depression symp- 
Table 4. Clinical characteristics related to the lateralization of epileptic focus and depression and anxiety symptoms of surgical patients with TLE

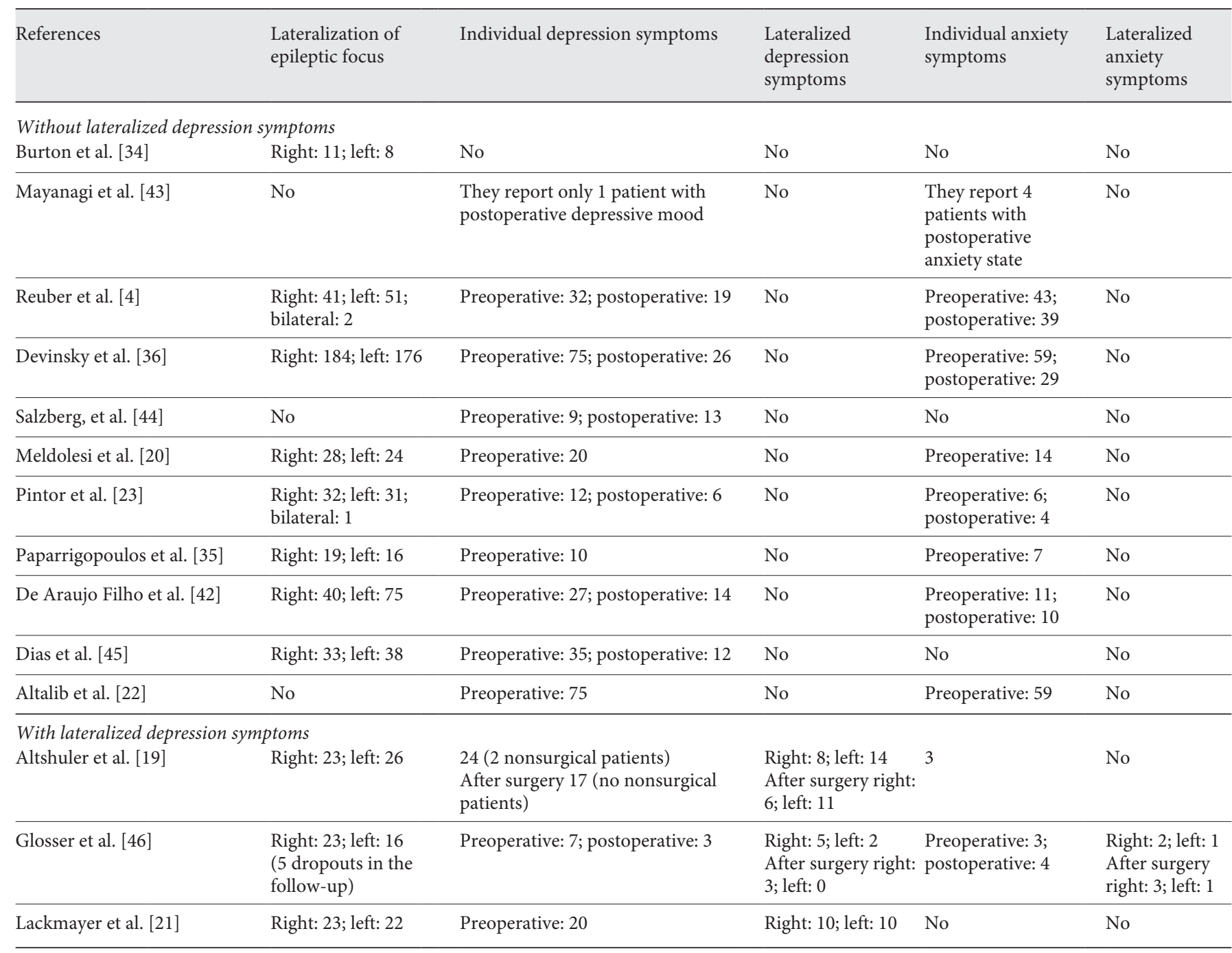

TLE, temporal lobe epilepsy.

toms post-surgery has been reported by some studies, especially when patients presented decreased seizure frequency [4]. Contrary to this finding, Meldolesi and colleagues [20] showed that though there was a reduction in anxiety symptoms after epilepsy surgery, depression persisted, even with the decline of seizure frequency in the majority of the patients. Studies also indicated that the diagnosis of depression prior to the surgery did not affect the surgical outcome for seizure control [21, 22]. However, Pintor et al. [23] could relate the prevalence of psychiatric symptoms before surgery with the occurrence of psychiatric conditions post-surgery. Furthermore, studies have highlighted the manifestation of anxiety in most of the epilepsy surgery candidates [24]. Anxiety can remain during the first year post-surgery [4] and can depend on the seizure control after surgery [25]. According to the literature on the subject, depression and cognitive dysfunction are not always associated with epilepsy. A part of population with the disease may not present cognitive or emotional impairment. These variables may depend on numerous factors such as frequency of crises, location and lateralization of the epileptic focus, other neurological pathology comorbid to the disease, and even the adequate control of the crises [26].

Though a number of treatments are available to reduce seizure frequency, its effect on depression or anxiety 
symptoms in epileptic patients should also be verified. The AEDs used for the treatment were not mentioned in most of the studies, considered for the present review. However, it is an important aspect since AEDs may be responsible for an increase or decrease in depression and anxiety symptoms in epileptic patients [27]. Moreover, some antidepressant drugs may contribute to the alteration of seizure frequency [28]. Besides, it is also important to report any change in the number of AEDs prescribed to the patients after surgery. Prayson and colleagues [29] demonstrated that the quantity of AEDs was reduced in only $28 \%$ of the patients after surgery, whereas $58 \%$ of the patients continued with the same AEDs after surgery. It is evidenced by the literature that the non-control of epileptic seizures directly impacts the patient's quality of life; thus, the higher the frequency/intensity of the seizures, the more prevalent are the desadaptive emotional symptoms. The prevalence of depression varies from 20 to $55 \%$ in patients with recurrent seizures and from 3 to $9 \%$ in patients with controlled seizures [30, 31].

In the present review, the majority of the evaluated studies have shown lateralization of epileptic focus occurred in the left hemisphere. However, no clear consensus is present regarding the association of depression lateralization and epileptic focus. Some studies depicted that depression occurs more frequently after lesions or seizure focus in the left hemisphere [32, 33]. According to Burton and Labar [34], increased depression was reported by patients who were subjected to left temporal lobectomy in comparison to the patients with right temporal lobectomy. Besides, a correlation between the severity of depression and the extension of hippocampus and amygdala resection, especially in left temporal lobectomy, was established by Paparrigopoulos et al. [35]. Furthermore, surgical laterality and location can be predisposing factors for psychological outcomes in epileptic patients post-surgery. Prayson et al. [29] elucidated more significant depression and anxiety symptoms among patients with left TLE before surgery as compared to the patients with left frontal lobe epilepsy. On the other hand, Devinsky et al. [36] demonstrated no association between the presence or absence of depression and anxiety and the lateralization or localization of the seizure onset before surgery. Besides, Manchanda et al. [24] also failed to establish any correlation between the laterality of seizure focus and depression symptoms. A study carried out in 2009 by Wrench et al. [37] compared the pre and postoperative depression scores between 2 groups of patients with epilepsy: with a mesial and non-mesial epileptogenic focus. The preoperative result indicates sim- ilarity in the depression scores of the 2 groups. However, a higher percentage of patients with mesial TLE showed symptoms of depression when compared to the group of non-mesials in the postoperative period [37]. The literature suggests that depression after surgery is associated with significantly smaller volumes of the hippocampus and amygdala contralateral to resection. Other factors, such as being free of crises after surgery and not having symptoms of depression in the previous period, predict a better outcome of the surgery in relation to emotional aspects $[38,39]$.

In this present systematic review, the evaluated data related to individual depressive diagnosis and anxiety, in both situations, no diagnosis was representative in preoperative and postoperative cases. Any kind of PD or neuropsychological evaluation was not executed by most of the evaluated studies in this review. The included studies that performed PD the primary method utilized was DSM/Structured Clinical Interview for DSM. Different methods of depression diagnosis may lead to distinct conclusions and create hurdles in generalizing the outcomes [40]. Besides, some studies have recognized the preoperative depression diagnosis as potential complications in the postoperative seizure outcome of patients with refractory epilepsy [41]. De Araujo Filho and co-authors [42] also demonstrated the absence of depression diagnosis preoperative or postoperative was associated with favorable seizure outcomes after surgery.

\section{Conclusions}

The prevalence of psychiatric disorders among refractory epileptic patients undergoing surgical procedures has been documented by various studies. However, no clear association has been elucidated, so far, between lateralization of seizure focus and the existence of depression and anxiety conditions. Surgery is considered the best treatment option for refractory epileptic patients. Determination of the risk of depression and anxiety symptoms in epileptic patients before surgical procedures is important to estimate the efficacy of surgery in the psychiatry episodes and its relation to seizure control. Besides, identification of these symptoms prior to surgery is vital to distinguish those patients who will require a longer specific psychological surveillance after surgery. Thus, rigorous preoperative and postoperative evaluation of psychiatry conditions is essential in patients with refractory epilepsy candidates for surgery. 


\section{Acknowledgements}

J.C.C. is funded by $\mathrm{CNPq}$ (research productivity scholarship). J.C.C. is supported by Conselho Nacional de Desenvolvimento Científico e Tecnológico - (CNPq) Brazil grant PQ 307372/20154. Our study was supported by the following grants: $\mathrm{CNPq}$ (Conselho Nacional de Desenvolvimento Científico e Tecnológico) and Coordenação de Aperfeiçoamento de Pessoal de Nível Superior (CAPES).

\section{Conflict of Interest Statement}

The authors have no conflict of interest to disclose.

\section{Funding Sources}

This research did not receive any other specific grant from funding agencies in the public, commercial, or not-for-profit sectors.

\section{Author Contributions}

Graciane Radaelli drafted the manuscript, evaluated and defined any disagreements between the groups that performed the literature search, and performed the statistical analysis. Fernanda Majolo performed the literature search. Eduardo Leal-Conceição critically reviewed the manuscript and statistics. Francisco de Souza Santos reviewed the methodological quality and data extraction. Vinícius Escobar reviewed the methodological quality and data extraction. Gabriele Goulart Zanirati critically reviewed the manuscript. Mirna Wetters Portugueza critically reviewed the manuscript. Fulvio Alexandre Scorza critically reviewed the manuscript. Jaderson Costa da Costa designed and coordinated the study and critically reviewed the manuscript.

\section{References}

1 Kwan P, Brodie MJ. Early identification of refractory epilepsy. N Engl J Med. 2000;342(5): 314-9.

2 Löscher W. How to explain multidrug resistance in epilepsy? Epilepsy Curr. 2005;5(3): $107-12$.

3 Blair RD. Temporal lobe epilepsy semiology. Epilepsy Res Treat. 2012;2012: 751510.2012

4 Reuber M, Andersen B, Elger CE, Helmstaedter C. Depression and anxiety before and after temporal lobe epilepsy surgery. Seizure. 2004;13(2):129-35.

5 Butler C, Zeman AZ. Neurological syndromes which can be mistaken for psychiatric conditions. J Neurol Neurosurg Psychiatry. 2005; 76(Suppl 1):i31-38

6 Sadler RM. The syndrome of mesial temporal lobe epilepsy with hippocampal sclerosis: clinical features and differential diagnosis. Adv Neurol. 2006;97:27-37.

7 Brandt C, Schoendienst M, Trentowska M, May TW, Pohlmann-Eden B, Tuschen-Caffier B, et al. Prevalence of anxiety disorders in patients with refractory focal epilepsy: a prospective clinic based survey. Epilepsy Behav. 2010;17(2):259-63.

8 Epps SA, Weinshenker D. Rhythm and blues: animal models of epilepsy and depression comorbidity. Biochem Pharmacol. 2013;85(2): $135-46$.

9 Manchanda R, Schaefer B, McLachlan RS, Blume WT. Interictal psychiatric morbidity and focus of epilepsy in treatment-refractory patients admitted to an epilepsy unit. Am J Psychiatry. 1992;149(8):1096-8.

10 Swinkels WA, van Emde Boas W, Kuyk J, Van Dyck R, Spinhoven P. Interictal depression, anxiety, personality traits, and psychological dissociation in patients with temporal lobe epilepsy (TLE) and extra-TLE. Epilepsia. 2006;47(12):2092-103.

11 Foong J, Flugel D. Psychiatric outcome of surgery for temporal lobe epilepsy and presurgical considerations. Epilepsy Res. 2007;75(23):84-96.

12 Mansouri A, Fallah A, Valiante TA. Determining surgical candidacy in temporal lobe epilepsy. Epilepsy Res Treat. 2012;2012: 706917.

13 Brooks J, Baker GA, Aldenkamp AP. The A-B neuropsychological assessment schedule (ABNAS): the further refinement of a patientbased scale of patient-perceived cognitive functioning. Epilepsy Res. 2001;43:227-37.

14 Kwon OY, Park SP. Depression and anxiety in people with epilepsy. J Clin Neurol. 2014; 10(3):175-88.

15 Higgins J, Thomas J. Cochrane handbook for systematic reviews of interventions. Chichester: John Wiley \& Sons; 2011.

16 Moher D, Liberati A, Tetzlaff J, Altman DG PRISMA Group. Preferred reporting items for systematic reviews and meta-analyses: the PRISMA statement. PLoS Med. 2009;6(7): e1000097.

17 Kellett MW, Smith DF, Baker GA, Chadwick DW. Quality of life after epilepsy surgery. J Neurol Neurosurg Psychiatry. 1997;63(1):52-8.

18 Alonso NB, Silva TId., Westphal-Guitti AC, Azevedo AM, Caboclo LOSF, Sakamoto AC, et al. Quality of life related to surgical treatment in patients with temporal lobe epilepsy due to mesial temporal sclerosis. J Epilepsy Clin Neurophysiol. 2006;12(4): 233-41

19 Altshuler LL, Devinsky O, Post RM, Theodore W. Depression, anxiety, and temporal lobe epilepsy: laterality of focus and symptoms. Arch Neurol. 1990;47:284-8.
20 Meldolesi GN, Di Gennaro G, Quarato PP, Esposito V, Grammaldo LG, Morosini P, et al Changes in depression, anxiety, anger, and personality after resective surgery for drugresistant temporal lobe epilepsy: a 2-year follow-up study. Epilepsy Res. 2007;77(1):2230.

21 Lackmayer K, Lehner-Baumgartner E, Pirker S, Czech T, Baumgartner C. Preoperative depressive symptoms are not predictors of postoperative seizure control in patients with mesial temporal lobe epilepsy and hippocampal sclerosis. Epilepsy Behav. 2013;26(1):81-6.

22 Altalib HH, Berg AT, Cong X, Vickrey BG, Sperling MR, Shinnar S, et al. Presurgical depression and anxiety are not associated with worse epilepsy surgery outcome five years postoperatively. Epilepsy Behav. 2018;83:712.

23 Pintor L, Bailles E, Fernández-Egea E, Sánchez-Gistau V, Torres X, Carreño M, et al. Psychiatric disorders in temporal lobe epilepsy patients over the first year after surgical treatment. Seizure. 2007;16(3):218-25.

24 Manchanda R, Schaefer B, McLachlan RS, Blume WT, Wiebe S, Girvin JP, et al. Psychiatric disorders in candidates for surgery for epilepsy. J Neurol Neurosurg Psychiatry. 1996;61(1):82-9.

25 Mattson P, Tibblin B, Kihlgreen M, Kumlien E. A prospective study of anxiety with respect to seizure outcome after epilepsy surgery. Seizure. 2005;14:40-5.

26 Ring HA, Acton PD, Scull D, Costa DC, Gacinovik S, Trimble MR. Patterns of brain activity in patients with epilepsy and depression. Seizure. 1999;8(7):390-7.

27 Mula M, Schmitz B. Depression in epilepsy: mechanisms and therapeutic approach. Ther Adv Neurol Disord. 2009;2(5):337-44. 
28 Cardamone L, Salzberg MR, O'Brien TJ, Jones NC. Antidepressant therapy in epilepsy: can treating the comorbidities affect the underlying disorder? Br J Pharmacol. 2013;168(7): 1531-54.

29 Prayson BE, Floden DP, Ferguson L, Kim KH, Jehi L, Busch RM. Effects of surgical side and site on psychological symptoms following epilepsy surgery in adults. Epilepsy Behav. 2017; 68:108-14.

30 Gilliam FG, Santos J, Vahle V, Carter J, Brown $\mathrm{K}$, Hecimovic H. Depression in epilepsy: ignoring clinical expression of neuronal network dysfunction? Epilepsia. 2004;45(Suppl 2):28-33.

31 Kandratavicius L, Hallak JEC, Leite JP. Psicose e depressão na epilepsia do lobo temporal. J Epilepsy Clin Neurophysiol. 2007;13(4): 163-7.

32 Davidson RJ. Parsing affective space: perspectives from neuropsychology and psychophysiology. Neuropsychology. 1993;7(4): $464-75$.
33 Mendez MF, Taylor JL, Doss RC, Salguero P. Depression in secondary epilepsy: relation to lesion laterality. J Neurol Neurosurg Psychiatry. 1994;57(2):232-3.

34 Burton LA, Labar D. Emotional status after right vs. left temporal lobectomy. Seizure. 1999;8(2):116-9.

35 Paparrigopoulos T, Ferentinos P, Brierley B, Shaw P, David AS. Relationship between post-operative depression/anxiety and hippocampal/amygdala volumes in temporal lobectomy for epilepsy. Epilepsy Res. 2008; 81(1):30-5.

36 Devinsky O, Barr WB, Vickrey BG, Berg AT, Bazil CW, Pacia SV, et al. Changes in depression and anxiety after resective surgery for epilepsy. Neurology. 2005;65(11):1744-9.

37 Wrench JM, Wilson SJ, Bladin PF, Reutens DC. Hippocampal volume and depression: insights from epilepsy surgery. J Neurol Neurosurg Psychiatry. 2009;80(5):539-44.
38 Devinsky O, Barr WB, Vickrey BG, Berg AT, Bazil CW, Pacia SV, et al. Changes in depression and anxiety after resective surgery for epilepsy. Neurology. 2005;65(11):1744-9.

39 Briellmann RS, Hopwood MJ, Jackson GD. Major depression in temporal lobe epilepsy with hippocampal sclerosis: clinical and imaging correlates. J Neurol Neurosurg Psychiatry. 2007;78(11):1226-30.

40 Fiest KM, Dykeman J, Patten SB, Wiebe S, Kaplan GG, Maxwell CJ, et al. Depression in epilepsy: a systematic review and meta-analysis. Neurology. 2013;80(6):590-9.

41 Kanner AM. Depression and epilepsy: a new perspective on two closely related disorders. Epilepsy Curr. 2006;6(5):141-6.

42 de Araújo Filho GM, Gomes FL, Mazetto L, Marinho MM, Tavares IM, Caboclo LO, et al. Major depressive disorder as a predictor of a worse seizure outcome one year after surgery in patients with temporal lobe epilepsy and mesial temporal sclerosis. Seizure. 2012; 21(8):619-23. 